\title{
A Novel Model Representation Framework for Cooperative Intelligent Transport Systems
}

\author{
Mohammed Obaid ${ }^{1 *}$, Zsolt Szalay ${ }^{1}$ \\ 1 Department of Automotive Technology, Faculty of Transportation Engineering and Vehicle Engineering, \\ Budapest University of Technology and Economics, H-1521 Budapest, P.O.B. 91, Hungary \\ * Corresponding author, e-mail: Obaid.mohammed@mail.bme.hu
}

Received: 21 January 2019, Accepted: 07 February 2019, Published online: 21 May 2019

\begin{abstract}
Cooperative Intelligent Transport Systems is C-ITS a set of ITS technologies that can provide services supported by the permanent, real time, information circulation among the components of the system. The paper aims to give an overview related to the modelling and evaluation possibilities of cooperative intelligent transportation system and to clarify the definition of the C-ITS and its differences from the regular ITS solutions. The paper introduce a proposed architecture of C-ITS modelling framework by describing C-ITS components, transferred data and its applications.
\end{abstract}

Keywords

C-ITS, modelling, architecture, framework

\section{Introduction}

The Intelligent transport systems (ITS) hopefully implements environment-friendly, safe and secured transportation processes (Tettamanti et al., 2016) that are supported by advanced technologies related to the fields of data detection, transmission and storage, communication, and control. Accordingly ITS is applied to increase safety (Alali et al., 2018), sustainability, efficiency, and comfort. Cooperative Intelligent Transport Systems (C-ITS) can be defined as the set of ITS technologies which can provide services supported by the permanent, real time, information circulation among the components of the system. For example C-ITS can make accident-related information available such as traffic conditions, unexpected events, and weather conditions to the driver in real time (De La Torre et al., 2018).

From a structural point of view the components of Intelligent Transport Systems (ITS) provide effective cooperation to support transportation processes applying digital technologies (Xue et al., 2014). These system components can be practically placed on the side of the road, directly in the vehicles (Schramm et al., 2018), at the transportation center or somewhere in the cloud (Rizviet et al., 2017). Summing up, C-ITS involves the cooperating components of the transportation processes including the users (Gratian et al., 2018), the vehicles, the infrastructure and the operator. (European Transport Safety Council, 2017)
The difference between ITS and C-ITS is that the interaction among the components (e.g. user, vehicle, infrastructure, environment, operator, etc.) of the system plays a prior role in case of the C-ITS (Zöldy, 2018), whilst in case of the ITS the process supporting intelligence can be implemented even in case of a specific system module (Guerrero-Higueras et al., 2018). C-ITS functions become more and more efficient with the increasing amount of exchanged information. It is important to understand that C-ITS is not a tool in its own, C-ITS is a combination of rules, procedures, systems and sub-systems to enable cooperative service delivery (Favarò et al., 2018).

Actors in a C-ITS framework can communicate with any other actor to send, receive and exchange information. Individual vehicle can talk with other vehicle (V2V) or roadside stations/ infrastructure (V2I). These communications provide safer, more comfortable traffic flow and lower emission from vehicles. Fig. 1 represents a simplified comparison of ITS and C-ITS.

Sun et al. (2016) describe C-ITS architecture and the numerous stakeholders needed to be considered during the development of such architecture. They classified the C-ITS application into two groups. The first class involves the solutions supporting environmental operation related issues and the second groups contain approaches reducing risk 

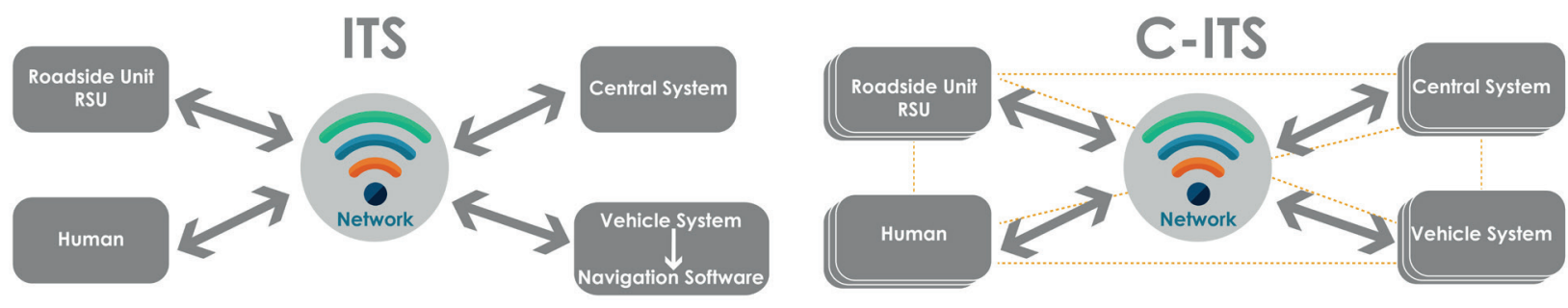

Fig. 1 ITS vs. C-ITS Systems

and increasing safety. Solodkiy and Yenokayev (2017) have studied C-ITS projects carried out in the European Union, especially focusing on the outcomes of the evaluated projects and their impact on road safety. Fouchal et al. (2017) have developed a framework that can be applied to evaluate and validate C-ITS systems (Mullins et al., 2018) the methodology takes into account all relevant C-ITS components (RSU, Vehicle, Control Centre) with their specific features.

Petrov et al. (2017) have presented the effect of C-ITS functions for a microscopic model affected by a traffic accident. The research has investigated both vehicle-to-vehicle (V2V) and vehicle-to-infrastructure (V2I) communication, especially focusing on computer simulation and communication modelling possibilities for current and future communication technologies (VANET, 4G, 5G). The outcome of the study has showed that a lower level congestion can be achieved at the intersection by the application of advanced communication technologies, since the arriving vehicles are able to modify their route by receiving the adequate information through $\mathrm{V} 2 \mathrm{~V}$ or V2I communication channels.

Ehlers et al. (2017) have analyzed the effect of C-ITS on network safety (Hajiagha et al., 2016). They suggested a bowtie analysis as a conceptual framework for evaluating the safety effect of C-ITS. Vinel et al. (2018) have addressed the performance of $\mathrm{V} 2 \mathrm{~V}$ communications and the performance of C-ITS safety applications. Their study has evaluated the case of Emergency Electronic Brake Lights (EEBL) where a braking vehicle send data packs to the following vehicle. This function can support the other vehicles in the safely performed braking process.

Summing up, the paper aims to give an overview related to the modelling and evaluation possibilities of cooperative intelligent transportation system (C-ITS). In the next section the basic methodology of C-ITS is presented which contains C-ITS components, transferred data and its application and a small description of C-ITS simulators. The third section presents a discussion about the introduced novel methodology and a small summary in the last section.

\section{Methodology}

This section presents a brief description of C-ITS components, types of transferred data / information properties, attributes and the application possibilities. The below presented description can help the reader in understanding C-ITS and its impact on safety and efficiency on road transport system.

\subsection{C-ITS Components}

Cooperative intelligent transportation system (C-ITS) consists of several components that work together to enhance safety (Ghadi et al., 2017) and operation efficiency. In general, three main C-ITS components are differentiated: On-Board Unit OBU (in-Vehicle) uses WAVE communication method to send and receive vehicle information (e.g. Location, status, operation) to Road Side Units (RSU) or nearby vehicles. The other one is the mentioned Road Side Units (RSU) that collects various information transmitted from OBUs, stores it and transmits it to the Transport Management Centre System (TMC) or send back information to vehicles (Ficco et al., 2017). The Third component is Transport Management Center System (TMC) which consists of a C-ITS server and a big data server to process large data (Todorovic et al., 2017). TMC processes the information using the data collected through the RSU and the supporting system (Automatic Incident Detection System, Pedestrian Detection System, Tolling System, Road Weather Information System, etc.) to provide the necessary information to the driver (Ding et al., 2018).

As described above some components of C-ITS operate as a supportive systems to collect other information and send it to RSU or TMC for processing. Automatic Incident Detection System is a support system that detects unpredicted situation (fallen objects, stationary vehicles...etc.) on the road and transmits it to TMC and RSU. Smart (WAVE) Tolling Support System is used in case of multi-lanes, non-stop toll roads through the installation of WAVE communication components. Pedestrian Detection System is also a support system that detects pedestrians, 
bicycles on the crosswalk area and transmits it to TMC and RSU. It can be either installed inside the vehicle or by using closed-circuit television cameras (CCTV) on roads. Road Weather Information System detects local weather characteristics and weather dependent road conditions and transfers it to the RSUs and the TMC. Traffic Signal Controller Support system controls traffic signal system in intersections such as signal phases and cycle duration in real time according to traffic condition. Fig. 2 shows a simplification of C-ITS system components.

\subsection{Transferred Data /Information and Its Applications}

Since from an operational point of view, real-time transportation processes are performed on the road (Petrillo et al., 2018), the vehicle related information exchange processes have a crucial significance in C-ITSs. Accordingly, the vehicle related communication processes are analyzed with a special attention. Two communication types are discussed: vehicle to vehicle communication (V2V) and vehicle to infrastructure communication (V2I). IEEE 802.11 WAVE standard, initiated from the allocation of the Dedicated Short Range Communications (DSRC) spectrum, provides a communication standard focusing on the channel between vehicles and infrastructure. However, there arise new innovative communication solutions almost every day. For example Bluetooth is a relatively simple communication technology and it can nearly found everywhere. Nowadays the main usage of Bluetooth related to the individual transportation to provide the conditions of a handsfree communication system that help road users to focus on the road. On the other hand, in V2I systems it can be used to provide communication channel between the vehicle and the traffic signal systems. WIFI and mobile (cellular) network technology such as GSM (Global System for Mobile

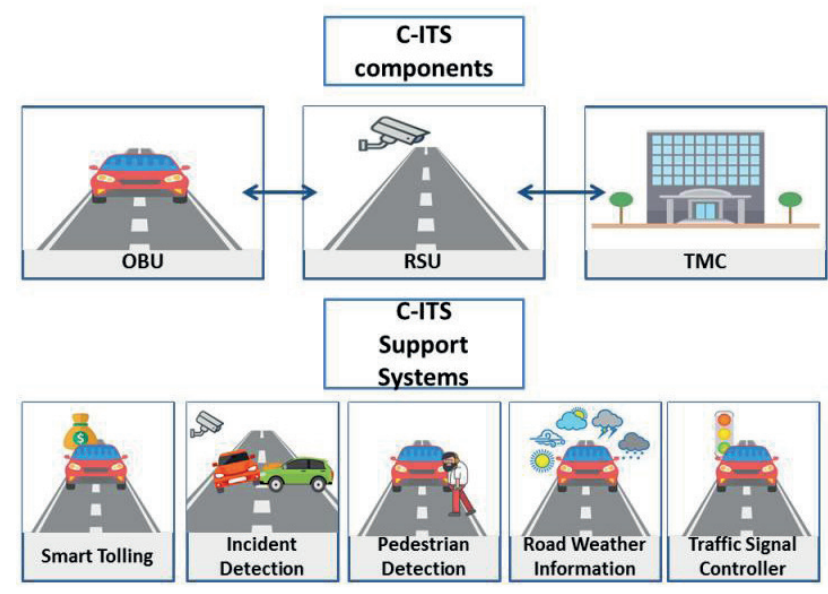

Fig. 2 C-ITS System Components communication) can provide a capable, secured (Ghanavi et al., 2018) and reliable communication (Gopalakrishnan et al., 2018) solutions for V2X applications.

$\mathrm{V} 2 \mathrm{~V}$ and V2I improve safety and efficiency through providing information to the driver. Safety applications can be applicable based on V2V communication. These applications can warn the driver, when he or she enters an intersection or leaves the highways. In case of a complex V2V communication system hazardous locations can also be prognosticated, accidents can be reported, sudden stops can be warned, conflicts can be sensed and pre-crash warning can be activated. V2I communication increases safety in intersection, supports speed management, rail crossing operations, priority for emergency vehicles. V2V and V2I improve efficiency of the transportation network by providing information for both the drivers on the network and the operator of the transportation network. By improving the efficiency of route planning and navigation (Esztergár et al., 2015), increasing the capacity of intelligent intersections, applying smart traffic lights, automated traffic intersection controls, traffic jam notifications and prior recognition of potential traffic jams a complex C-ITS framework can significantly support the technical and economic objectives of the modern society (El Mrabet et al., 2018).

\subsection{Simulation}

The so called Vehicular Ad-Hoc Networks or VANETs represent a sub form of Mobile Ad-Hoc Networks or MANETs that provide communication between vehicles and between vehicles and other system components (e.g. RSUs deployed on the infrastructure, mobile components like drones, etc.) to support the efficient and safe transportation (Chadha, 2015). The high mobility of nodes and their behavioral patterns in a VANET networks require more realistic simulation to get reliable results, close to real life conditions (Mascareñas et al., 2017). This can be archived by combining traffic and network simulation software to perform proper cooperation.

Traffic simulation solutions are software used mainly by transportation experts to simulate various real life traffic flow conditions. Traffic simulation models can be used in VANET simulation as mobility data generators providing input for the network simulation application. Table 1 summarizes the most relevant traffic simulation model and their properties:

Network simulation models are used for simulation of numerous parameters of network traffic to describe the 
Table 1 Traffic Simulator Properties

\begin{tabular}{lcccc}
\hline Simulator & Cost & Model & $\begin{array}{c}\text { User-defined } \\
\text { maps }\end{array}$ & $\begin{array}{c}\text { Source Code } \\
\text { Availability }\end{array}$ \\
\hline SUMO & Free & $\begin{array}{l}\text { Micro/ } \\
\text { Macro }\end{array}$ & Yes & Open \\
FreeSim & Free & Micro & No & Open \\
CityMob & Free & Micro & Yes & Open \\
Vissim & Commercial & Micro & Yes & Closed \\
Visum & Commercial & Macro & Yes & Closed \\
\hline
\end{tabular}

network behavior under various circumstances. They can also be used to optimize network topologies and communication procedures. The most known network simulation models for VANET are the OPNET, the GloMoSim, the SNS and the OMNET++.

\section{Discussion}

Based on the classification of C-ITS components, transferred data and its application possibilities it can be seen that C-ITS can provide a common framework to harmonize the operation of different system components to support a safer, a more secured (Khalid et al., 2018), a more efficient and a more reliable transport service for road users. Fig. 3 represents the C-ITS architecture.

C-ITS system improves both road safety and traffic flow efficiency at both microscopic and macroscopic level. The question is that how much the presented individual system components can improve network efficiency in case of separated operation or in case of a cooperative operation;

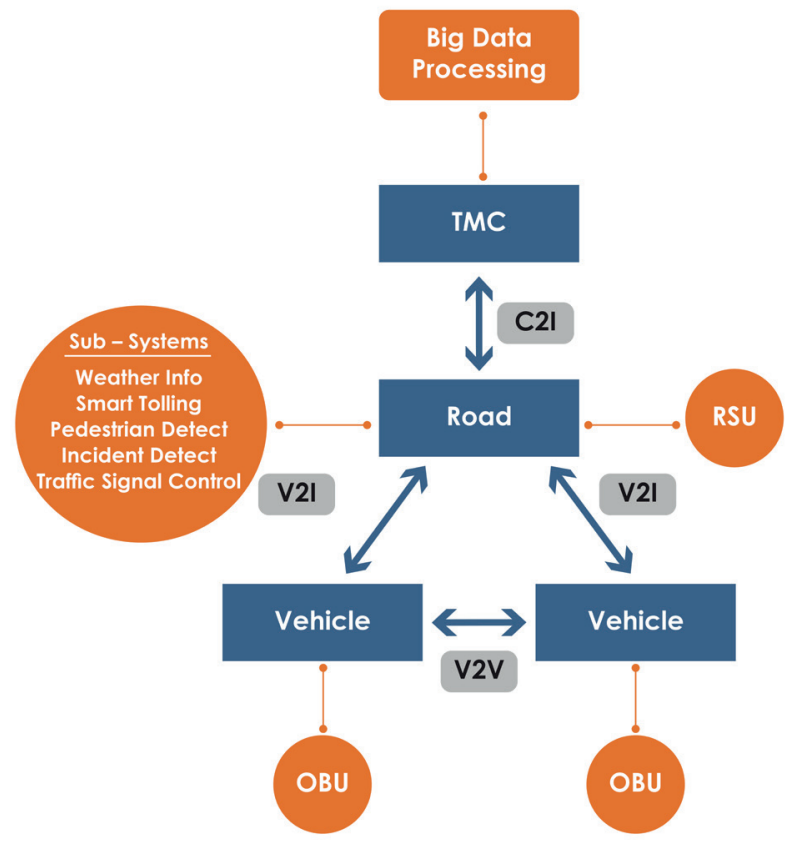

Fig. 3 C-ITS system Architecture for example what is the effect of transmitting traffic related data among the vehicles especially in case of congested environment or sending warning signs to the road users in case of an accident. The previous section the most relevant traffic and network simulation model gave been introduced that can be used for microscopic and macroscopic simulation. By using a combination of the presented models, the effect of C-ITS system on the network can be estimated. To design the framework which is applicable to study the mentioned effect, the paper introduces the proposed model approach in the following section. The first step is to represent C-ITS system using VANET in the microscopic fragment of the whole network and perform complex a scenario analysis to this model framework using traffic and network simulation models. The results can be used as an input for the macroscopic model to estimate the effect of the chosen C-ITS application in case of the whole network. In the next step the macroscopic model results can be used to improve the microscopic model. The introduced framework is shown in Fig. 4.

\section{Conclusion}

C-ITS is a combination of technologies that cooperate with each other to provide a safe transportation system, and efficiently share reliable, real time information related to the transportation processes among the users. Accordingly, this paper gives an overview about a proposed C-ITS system framework which makes it possible to describe the effect of the different cooperative technologies on the efficiency of transportation processes.

The paper also clarifies the definition of the C-ITS and its differences from the regular ITS solutions. In the literature review some relevant researches related to C-ITS have been presented. The second part of the paper focuses

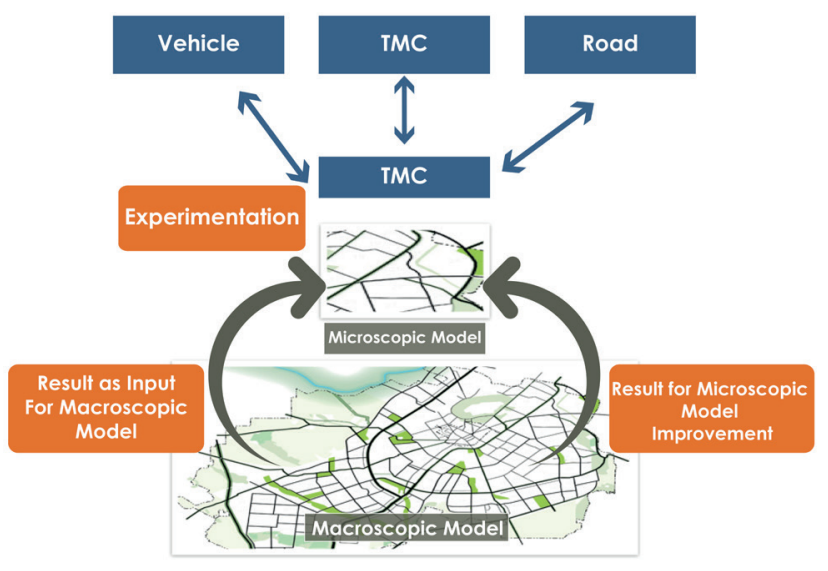

Fig. 4 C-ITS Modelling Framework 
on C-ITS components, how these components communicate with each other and the expected effect of the cooperation. The proposed architecture of C-ITS modelling framework has been introduced using the described components, transferred data and its applications.

\section{References}

Alali, M., Almogren, A., Hassan, M. M., Rassan, I. A., Bhuiyan, M. Z. A. (2018) "Improving risk assessment model of cyber security using fuzzy logic inference system", Computers \& Security, 74, pp. 323-339. https://doi.org/10.1016/j.cose.2017.09.011

Chadha, D. (2015) "Vehicular Ad hoc Network (VANETs): A Review", International Journal of Innovative Research in Computer and Communication Engineering, 3(3), pp. 2339-2346.

De La Torre, G., Rad, P., Choo, K. K. R. (2018) "Driverless vehicle security: Challenges and future research opportunities", Future Generation Computer Systems. https://doi.org/10.1016/j.future.2017.12.041

Ding, D., Han, Q. L., Xiang, Y., Ge, X., Zhang, X. M. (2018) "A survey on security control and attack detection for industrial cyber-physical systems", Neurocomputing, 275, pp. 1674-1683. https://doi.org/10.1016/j.neucom.2017.10.009

Ehlers, U. C., Ryeng, E. O., McCormack, E., Khan, F., Ehlers, S. (2017) "Assessing the safety effects of cooperative intelligent transport systems: A bowtie analysis approach", Accident Analysis and Prevention, 99, pp. 125-141. https://doi.org/10.1016/j.aap.2016.11.014

El Mrabet, Z., Kaabouch, N., El Ghazi, H., El Ghazi, H. (2018) "Cybersecurity in smart grid: Survey and challenges", Computers \& Electrical Engineering, 67, pp. 469-482. https://doi.org/10.1016/j.compeleceng.2018.01.015

Esztergár-Kiss, D., Csiszár, C. (2015) "Evaluation of multimodal journey planners and definition of service levels", International journal of intelligent transportation systems research, 13(3), pp. 154-165. https://doi.org/10.1007/s13177-014-0093-0

European Transport Safety Council (2017) ETSC November 2017, BRIEFING Cooperative Intelligent Transport Systems (C-ITS).

Favarò, F., Eurich, S., Nader, N. (2018) "Autonomous vehicles' disengagements: trends, triggers, and regulatory limitations", Accident Analysis \& Prevention, 110, pp. 136-148. https://doi.org/10.1016/j.aap.2017.11.001

Ficco, M., Palmieri, F. (2017) "Security and Resilience in Intelligent Data-Centric Systems and Communication Networks", 1st ed, Academic Press, Massachusetts, USA. https://doi.org/10.1016/c2016-0-01083-5

Fouchal, H., Bourdy, E., Wilhelm. G Ayaida, M. (2017) "A validation tool for cooperative intelligent transport systems", Journal of Computational Science, 22, pp. 283-288. https://doi.org/10.1016/j.jocs.2017.05.026

Ghanavi, R., Kalantari, E., Sabbaghian, M., Yanikomeroglu, H., Yongacoglu, A. (2018) "Efficient 3D aerial base station placement considering users mobility by reinforcement learning", In: Wireless Communications and Networking Conference (WCNC), IEEE, Barcelona, Spain, pp. 1-6. https://doi.org/10.1109/wcnc.2018.8377340
Traffic and network simulation models can be used together to achieve similar results to the case of assuming real life VANET communication processes. These simulation models can describe the effect of C-ITS at both microscopic and macroscopic levels.

Ghadi, M., Török, Á., Tánczos, K. (2017) "Integration of Probability and Clustering Based Approaches in the Field of Black Spot Identification", Periodica Polytechnica Civil Engineering, 63(1), pp. 46-52.

https://doi.org/10.3311/ppci.11753

Gopalakrishnan, K., Gholami, H., Vidyadharan, A., Choudhary, A., Agrawal, A. (2018) "Crack damage detection in unmanned aerial vehicle images of civil infrastructure using pre-trained deep learning model", International Journal for Traffic and Transport Engineering, 8(1), pp. 1-14. https://doi.org/10.7708/ijtte.2018.8(1).01

Gratian, M., Bandi, S., Cukier, M., Dykstra, J., Ginther, A. (2018) "Correlating human traits and cyber security behaviour intentions", Computers \& Security, 73, pp. 345-358. https://doi.org/10.1016/j.cose.2017.11.015

Guerrero-Higueras, Á. M., DeCastro-Garcia, N., Matellan, V. (2018) "Detection of Cyber-attacks to indoor real time localization systems for autonomous robots", Robotics and Autonomous Systems, 99 , pp. $75-83$.

https://doi.org/10.1016/j.robot.2017.10.006

Hajiagha, S. H. R., Hashemi, S. S., Mohammadi, Y., Zavadskas, E. K. (2016) "Fuzzy belief structure based VIKOR method: an application for ranking delay causes of Tehran metro system by FMEA criteria", Transport, 31(1), pp. 108-118. https://doi.org/10.3846/16484142.2016.1133454

Khalid, A., Kirisci, P., Khan, Z. H., Ghrairi, Z., Thoben, K. D., Pannek, J. (2018) "Security framework for industrial collaborative robotic cyber-physical systems", Computers in Industry, 97, pp. 132-145. https://doi.org/10.1016/j.compind.2018.02.009

Mascareñas, D. D., Stull, C. J., Farrar, C. R. (2017) "Autonomous execution of the Precision Immobilization Technique", Mechanical Systems and Signal Processing, 87, pp. 153-168. https://doi.org/10.1016/j.ymssp.2016.06.043

Mullins, G. E., Stankiewicz, P. G., Hawthorne, R. C., Gupta, S. K. (2018) "Adaptive generation of challenging scenarios for testing and evaluation of autonomous vehicles", Journal of Systems and Software, 137, pp. 197-215. https://doi.org/10.1016/j.jss.2017.10.031

Petrillo, A., Pescapé, A., Santini, S. (2018) "A collaborative approach for improving the security of vehicular scenarios: The case of platooning", Computer Communications, 122, pp. 59-75. https://doi.org/10.1016/j.comcom.2018.03.014

Petrov, T. Dado, M. Ambrosch K. E. (2017) "Computer Modelling of Cooperative Intelligent Transportation Systems", Procedia Engineering, 192, pp. 683-688. https://doi.org/10.1016/j.proeng.2017.06.118

Rizvi, S., Willet, J., Perino, D., Marasco, S., Condo, C. (2017) "A Threat to Vehicular Cyber Security and the Urgency for Correction", Procedia Computer Science, 114, pp. 100-105. https://doi.org/10.1016/j.procs.2017.09.021 
Schramm, D., Hiller, M., Bardini, R. (2018) "Single track models", In: Vehicle Dynamics Springer, Berlin, Heidelberg, Germany, pp. $225-257$.

https://doi.org/10.1007/978-3-540-36045-2_10

Solodkiy, A. Yenokayev, V. (2017) "Cooperative ITS — a Strategic Way to Ensure Road Safety", Transportation Research Procedia, 20, pp. $630-634$

https://doi.org/10.1016/j.trpro.2017.01.102

Sun, L., Li, Y., Gao, J. (2016) "Architecture and Application Research of Cooperative Intelligent Transport Systems", Procedia Engineering, 137, pp. 747-753.

https://doi.org/10.1016/j.proeng.2016.01.312

Tettamanti, T., Varga, I., Szalay, Z. (2016) "Impacts of autonomous cars from a traffic engineering perspective", Periodica Polytechnica Transportation Engineering, 44(4), pp. 244-250.

https://doi.org/10.3311/PPtr.9464
Todorovic, M., Simic, M., Kumar, A. (2017) "Managing transition to electrical and autonomous vehicles", Procedia Computer Science, 112, pp. 2335-2344. https://doi.org/10.1016/j.procs.2017.08.201

Vinel, A., Lyamin, N. Isachenkov, P. (2018) "Modelling of V2V Communications for C-ITS Safety Applications: a CPS Perspective", IEEE Communications Letters, 22(8), pp. 1600-1603. https://doi.org/10.1109/lcomm.2018.2835484

Xue, M., Wang, W., Roy, S. (2014) "Security concepts for the dynamics of autonomous vehicle networks", Automatica, 50(3), pp. 852-857. https://doi.org/10.1016/j.automatica.2013.12.001

Zöldy, M. (2018) "Legal Barriers of Utilization of Autonomous Vehicles as Part of Green Mobility", Proceedings in Automotive Engineering, pp. 243-248.

https://doi.org/10.1007/978-3-319-94409-8_29 